\title{
ZERO ENERGY ASYMPTOTICS OF THE RESOLVENT IN THE LONG RANGE CASE
}

\author{
S. FOURNAIS AND E. SKIBSTED
}

\begin{abstract}
We present a limiting absorption principle at zero energy for two-body Schrödinger operators with a long-range potential having a positive virial at infinity. Furthermore, we prove existence of limits (in weighted spaces), as the spectral parameter tends to zero, of all powers of the resolvent. The principal tools of proof are absence of eigenvalue at zero, singular Mourre theory and microlocal estimates. Some elements of the proof will be explained.
\end{abstract}

\section{Statement of MAin Results}

We give an account of some recent results on asymptotic expansion at zero of the resolvent $R(\zeta)=(H-\zeta)^{-1}$ of a two-body Schrödinger operator $H=-\Delta+V$ on $L^{2}\left(\mathbb{R}^{d}\right)$; see [5] for details. It is well-known, see [18], [12] and the more recent work [13] in which further references can be found, that if $V(x)=O\left(|x|^{-(2+\epsilon)}\right)$ with $\epsilon>0$ then such an asymptotic expansion exists. For the 'long-range' case, $V(x)=O\left(|x|^{-\mu}\right)$ with $\mu<2$, much less is known. To our knowledge, the only results on limiting absorption principles for such potentials are [22] and [17] (and [2] for the purely Coulombic case). In [22] only radially symmetric potentials are treated, and though radial symmetry is not imposed in [17] some of the assumptions of that paper appear unnecessarily restrictive. Here we present a complete asymptotic expansion of the resolvent at zero energy, for a much wider class of potentials. Our basic assumption is a sign condition at infinity,

$$
V(x) \leq-\epsilon|x|^{-\mu} ;|x|>R
$$

and a similar positive virial condition.

For such potentials we prove complete asymptotic expansions (in weighted spaces)

$$
R(\lambda+(-) i 0) \asymp \sum_{j=0}^{\infty} R_{j}^{+(-)} \lambda^{j} \text { for } \lambda \rightarrow 0^{+} ;
$$

INVITED TALK, ICMP LISBON, 2003 
here $R_{0}^{+} \neq R_{0}^{-}$. We also show that zero is not an eigenvalue. (This is implicit in (1.2).) We notice that there is no explicit dimensiondependence or fractional/inverse powers in $\lambda$.

It is well-known that for 'long-range' potentials that are positive at infinity, zero can indeed be an eigenvalue. This explains one aspect of the condition (1.1). Probably the best intuitive explanation of the result (1.2) is given in terms of the WKB-ansatz for stationary solutions to the Schrödinger equation $-\psi^{\prime \prime}+V \psi=E \psi$ in dimension $d=1$

$$
\psi \approx C_{+}(E-V)^{-\frac{1}{4}} e^{i \int(E-V)^{\frac{1}{2}} d x}+C_{-}(E-V)^{-\frac{1}{4}} e^{-i \int(E-V)^{\frac{1}{2}} d x} .
$$

Under the condition (1.1) the oscillatory behaviour survives for $E \approx 0$, with $E>0$ (since $\int(-V)^{\frac{1}{2}} d x \sim|x|^{1-\frac{\mu}{2}} \rightarrow \infty$ ). Moreover, (1.3) suggests that zero is not an eigenvalue, and also indicates which weights one needs in (1.2). We remark that indeed (1.2) can be proved for $d=1$ by WKB-methods, see [22], which can also be used to prove optimality of the weights in the results below.

Let us state our main results precisely. Let $0<\theta<\pi$ and define

$$
\Gamma_{\theta}=\{z \in \mathbb{C} \backslash\{0\}|| z \mid \leq 1, \arg z \in(0, \theta)\} .
$$

We have the following limiting absorption principle at zero energy for a Schrödinger operator $H=-\Delta+V$ on $\mathcal{H}=L^{2}\left(\mathbb{R}^{d}\right)$ recalling the notation $R(\zeta)=(H-\zeta)^{-1}$. Although we shall not elaborate here, it is enough to impose the conditions (1) and (3) near infinity.

Theorem 1.1. Let $V(x)=V_{1}(x)+V_{2}(x), x \in \mathbb{R}^{d}$, be a real-valued potential. Suppose there exists $0<\mu<2$ such that $V$ satisfies the conditions (1)-(6) below.

(1) There exists $\epsilon_{1}>0$ such that $V_{1}(x) \leq-\epsilon_{1}\langle x\rangle^{-\mu} ;\langle x\rangle=\sqrt{1+x^{2}}$.

(2) For all $\alpha \in(\mathbb{N} \cup\{0\})^{d}$ there exists $C_{\alpha}>0$ such that

$$
\langle x\rangle^{\mu+|\alpha|}\left|\partial^{\alpha} V_{1}(x)\right| \leq C_{\alpha} .
$$

(3) There exists $\epsilon_{2}>0$ such that $-|x|^{-2}\left(x \cdot \nabla\left(|x|^{2} V_{1}\right)\right) \geq-\epsilon_{2} V_{1}$.

(4) $V_{2}(-\Delta+i)^{-1}$ is a compact operator on $L^{2}\left(\mathbb{R}^{d}\right)$.

(5) There exists $\delta, C, R>0$ such that

$$
\left|V_{2}(x)\right| \leq C|x|^{-1-\mu / 2-\delta}
$$

for $|x|>R$.

(6) $V$ satisfies unique continuation at infinity (see Assumption 2.1 in Section 2).

Then for all $s \in\left(\frac{1}{2}+\frac{\mu}{4}, \frac{1}{2}+\frac{\mu}{4}+\delta\right)$ and all $0<\theta<\pi$ the family of operators $B(\zeta)=\langle x\rangle^{-s} R(\zeta)\langle x\rangle^{-s}$ is uniformly Hölder continuous in 
$\Gamma_{\theta}$. In particular there exists $C_{s, \theta}>0$ such that

$$
\sup _{\zeta \in \Gamma_{\theta}}\left\|\langle x\rangle^{-s} R(\zeta)\langle x\rangle^{-s}\right\| \leq C_{s, \theta},
$$

and the limits

$$
\begin{aligned}
\langle x\rangle^{-s} R(0+i 0)\langle x\rangle^{-s} & \equiv \lim _{\zeta \rightarrow 0, \zeta \in \Gamma_{\theta}}\langle x\rangle^{-s} R(\zeta)\langle x\rangle^{-s}, \\
\langle x\rangle^{-s} R(0-i 0)\langle x\rangle^{-s} & \equiv \lim _{\zeta \rightarrow 0, \zeta \in \Gamma_{\theta}}\langle x\rangle^{-s} R(\bar{\zeta})\langle x\rangle^{-s}
\end{aligned}
$$

exist in $\mathcal{B}\left(L^{2}\left(\mathbb{R}^{d}\right)\right)$.

Next, we have existence of limits for powers of the resolvent. The asymptotic expansion (1.2) is an easy consequence of Theorem 1.2 below. Notice also that Theorem 1.1 is a particular case of Theorem 1.2.

Theorem 1.2. Let $V=V_{1}+V_{2}$ satisfy the conditions in Theorem 1.1 with (5) replaced by: For some $m_{0} \in \mathbb{N}$

$\left(^{\prime}\right) V_{2}=O\left(k^{-m_{0}-\epsilon}\right) ; k=k(x)=\langle x\rangle^{1+\mu / 2}$.

Let $m \leq m_{0}, \theta \in(0, \pi)$ and $\epsilon>0$. Then there exists $C>0$ such that

$$
\left\|k^{-(m-1 / 2)-\epsilon} R(\zeta)^{m} k^{-(m-1 / 2)-\epsilon}\right\| \leq C,
$$

for all $\zeta \in \Gamma_{\theta}$.

Furthermore, the function

$$
\zeta \mapsto k^{-(m-1 / 2)-\epsilon} R(\zeta)^{m} k^{-(m-1 / 2)-\epsilon}
$$

is uniformly Hölder continuous in $\Gamma_{\theta}$.

Using Theorem 1.1 one may define (with $\mathcal{H}_{1}=k^{-1 / 2-\epsilon} L^{2}\left(\mathbb{R}^{d}\right), \mathcal{H}_{2}=$ $\left.k^{1 / 2+\epsilon} L^{2}\left(\mathbb{R}^{d}\right)\right)$ :

$$
E^{\prime}(+0)=(2 \pi i)^{-1}\{R(0+i 0)-R(0-i 0)\} \in \mathcal{B}\left(\mathcal{H}_{1}, \mathcal{H}_{2}\right) .
$$

One can prove that indeed

$$
E^{\prime}(+0) \neq 0
$$

Let $F(|x|<C)$ denote the multiplication operator by the characteristic function of $\{x|| x \mid<C\}$ and let $\kappa=(1+\mu / 2)^{-1}$.

Corollary 1.3. Under the conditions of Theorem 1.2 with (5') valid for all $m_{0} \in \mathbb{N}$ :

(i) For all $s>\frac{5}{2}\left(1+\frac{\mu}{2}\right)$ and $f \in C_{0}^{\infty}(\mathbb{R})$

$$
\left\|\langle x\rangle^{-s}\left(e^{-i t H}\left(f 1_{[0, \infty)}\right)(H)+i t^{-1} f(0) E^{\prime}(+0)\right)\langle x\rangle^{-s}\right\|=O\left(t^{-2}\right) .
$$


(ii) For all $0 \leq \epsilon^{\prime}<\epsilon \leq 1$ there exists $s>1$ (depending on $\epsilon$ ) such that for all $f \in C_{0}^{\infty}(\mathbb{R})$

$$
\left\|F\left(|x|<t^{(1-\epsilon) \kappa}\right) e^{-i t H}\left(f 1_{[0, \infty)}\right)(H)\langle x\rangle^{-s}\right\|=O\left(t^{-\left(1+\epsilon^{\prime}\right) \frac{1}{2}}\right) .
$$

Remark 1.4. 1) By time reversal invariance there are similar bounds for $t \rightarrow-\infty$.

2) Due to (1.7) and Corollary 1.3 (i) the best one could hope for to the right in $(1.8)$ would be the bound $O\left(t^{-1}\right)$ (for $f(0) \neq 0$ ). Moreover we would expect that $t^{\kappa}$ is indeed the borderline for this kind of low energy, minimal velocity estimate. In fact there is a sharp analogous bound in classical mechanics, cf. [6] and [20].

3) If $V_{2} \in C_{0}^{\infty}\left(\mathbb{R}^{d}\right)$ one may take $f=1$ in Corollary 1.3 (i) and (ii). This follows readily from the given statements and well-established high energy estimates, see [15, Theorem 1.1], [3, Theorem 1] or [11, Theorem 1.2 (ii)], in fact some local singularities may be included.

We shall outline the proof of Theorems 1.1 and 1.2 in the following sections. Apart from the notation $\langle x\rangle=\sqrt{1+x^{2}}$, used above, we will also need the notation $p=-i \nabla$ and $A=(x \cdot p+p \cdot x) / 2$.

The virial $W$ of the potential $V$ is defined by $W=-2 V-x \cdot \nabla V$. We recall the (formal) identity $i[H, A]=2 H+W$. By design of the splitting of $V$, the assumptions (1) and (3) of Theorem 1.1 yield

$$
W_{1}(x)=-2 V_{1}(x)-x \cdot \nabla V_{1}(x) \geq \epsilon_{1} \epsilon_{2}\langle x\rangle^{-\mu},
$$

so in particular, the virial $W$ is positive in the case $V_{2}=0$.

\section{AbSence of BOUnd StATES FOR SchröDinger OPERATORS}

In this section we present a basic result of independent interest, namely the absence of zero-energy bound states for long range potentials negative at infinity. The proof is a variation of the technique applied in the proof of [19, Theorem XIII.58] of which the present result is a generalization.

The conditions which exclude zero-energy eigenfunctions are given in Assumptions 2.1 and 2.2 below. Notice that the assumptions in Theorem 1.1 are stronger than Assumption 2.2: Take $h=\epsilon r^{-\mu / 2}$ for a small $\epsilon>0$ and $s$ close to 1 . Let us specify the notation $x=r \omega \in \mathbb{R}^{d}$, with $\omega \in \mathbb{S}^{d-1}$.

Assumption 2.1. The function $V: \mathbb{R}^{d} \rightarrow \mathbb{R}$ is measurable, and if $u \in H^{2}\left(\mathbb{R}^{d}\right), u=0$ in a neighbourhood of $\infty$, the product $V \psi \in L^{2}\left(\mathbb{R}^{d}\right)$ and $u$ is a distributional solution to

$$
-\Delta u+V u=0
$$


then $u=0$.

We remark that for $d \geq 3$ the condition $V \in L_{\text {loc }}^{d / 2}\left(\mathbb{R}^{d}\right)$ suffices, see [14].

Assumption 2.2. The function $V$ can be written as $V=V_{1}+V_{2}$, such that: For some $s \in[0,1)$, some $R, C>0$ and a positive differentiable function $h=h(r)$ defined on $[R, \infty)$ we have

(1) $V_{1}$ and $V_{2}$ are bounded on $|x|>R$, and $V_{1}$ is negative on $|x|>R$.

(2) $\sup _{\omega \in S^{d-1}} \frac{d}{d r}\left(r^{s+1} V_{1}(r \omega)\right) \leq-r^{s} h^{2}(r)$ when $r>R$.

(3) $r^{-1}+r \sup _{\omega \in S^{d-1}}\left|V_{2}(r \omega)\right|=o(h)$ as $r \rightarrow \infty$.

(4) $h^{\prime}(r) \leq C h^{2}(r)$ on $|x|>R$.

With the above assumptions we can prove the absence of zero-energy eigenstates.

Theorem 2.3. Suppose $V=V_{1}+V_{2}$ satisfies Assumptions 2.1 and 2.2. Suppose furthermore that $\psi \in H_{\mathrm{loc}}^{2}\left(\mathbb{R}^{d}\right)$ satisfies (1)-(3) below.

(1) $\int_{|x|>R} h^{2}(r)|\psi(x)|^{2} d x<\infty$ and $\int_{|x|>R}\left|V_{1}(x)\right||\psi(x)|^{2} d x<\infty$.

(2) $p_{j} \psi \in L^{2}\left(\mathbb{R}^{d}\right) ; j=1, \cdots, d$.

(3) The product $V \psi \in L_{\mathrm{loc}}^{2}\left(\mathbb{R}^{d}\right)$, and $(-\Delta+V) \psi=0$ in the sense of distributions.

Then $\psi=0$.

\section{ExTENDED LIMITING ABSORPTION PRINCIPLES}

We introduce that following symbols:

$$
\begin{gathered}
a_{0}(x, \xi)=f_{E}(x)^{-2} \xi^{2}, \quad b(x, \xi)=\frac{x}{\langle x\rangle} \cdot \frac{\xi}{f_{E}(x)}, \\
\text { with } \quad f=f_{E}=\sqrt{\kappa_{0}^{-2} E+(1-\mu / 2)^{-1}\langle x\rangle-\mu} ; \kappa_{0}, E>0,
\end{gathered}
$$

and where the parameters will be specified below.

Let us denote by $\mathrm{Op}^{\mathrm{w}}(a)$ the Weyl quantization of a symbol $a$. Explicitly $\mathrm{Op}^{\mathrm{w}}(a)$ acts as follows

$$
\left(\mathrm{Op}^{\mathrm{w}}(a) \phi\right)(x)=(2 \pi)^{-d} \iint e^{i(x-y) \xi} a((x+y) / 2, \xi) \phi(y) d y d \xi .
$$

Theorem 3.1. Let $V(x)$ satisfy the conditions of Theorem 1.1 with $V_{2}=0$. We reformulate the assumption (3) as: For some $\kappa_{0}>0$ and $2>\mu>0$,

$$
W(x)=-2 V(x)-x \cdot \nabla V(x) \geq 2 \kappa_{0}^{2}\langle x\rangle^{-\mu} .
$$

Let $\theta \in(0, \pi)$ and $\Gamma_{\theta}$ be as defined in (1.4). Let $a_{0}$ and $b$ be as defined in (3.1) with $E=|\zeta|$. Define $k=k(x)=\langle x\rangle^{1+\mu / 2}$. Then the following 
conclusions, (i) - (iv), hold for $H=p^{2}+V$ with all bounds being uniform in $\zeta \in \Gamma_{\theta}$ :

(i) Let $m \in \mathbb{N}$ and let $\epsilon>0$ be arbitrary. Then there exists $C>0$ such that

$$
\left\|k^{-(m-1 / 2)-\epsilon} R(\zeta)^{m} k^{-(m-1 / 2)-\epsilon}\right\| \leq C .
$$

(ii) There exists $C_{0}>0$, depending only on $V$, such that if $\operatorname{supp}\left(F_{+}\right) \subset$ $\left(C_{0}, \infty\right)$ and $F_{+}^{\prime} \in C_{0}^{\infty}(\mathbb{R})$, then for all $m \in \mathbb{N}$ and all $\epsilon, t>0$ there exists $C>0$ such that

$$
\begin{aligned}
& \left\|k^{t-1 / 2-\epsilon} \operatorname{Op}^{\mathrm{w}}\left(F_{+}\left(a_{0}\right)\right) R(\zeta)^{m} k^{-t-m+1 / 2-\epsilon}\right\| \leq C, \\
& \left\|k^{-t-m+1 / 2-\epsilon} R(\zeta)^{m} \mathrm{Op}^{\mathrm{w}}\left(F_{+}\left(a_{0}\right)\right) k^{t-1 / 2-\epsilon}\right\| \leq C .
\end{aligned}
$$

(iii) Let $\tilde{F}_{+}, \tilde{F}_{-}$satisfy (with $\kappa_{0}$ from (3.3)) for some $\kappa>0$,

- $\inf \operatorname{supp}\left(\tilde{F}_{+}\right)>-\kappa>-\kappa_{0}, \sup \operatorname{supp}\left(\tilde{F}_{-}\right)<\kappa<\kappa_{0}$.

- $\tilde{F}_{-}^{\prime}, \tilde{F}_{+}^{\prime} \in C_{0}^{\infty}(\mathbb{R})$.

Let $F_{-} \in C_{0}^{\infty}(\mathbb{R})$. Then for all $m \in \mathbb{N}$ and all $\epsilon, t>0$ there exists $C>0$ such that

$$
\begin{aligned}
& \left\|k^{t-1 / 2-\epsilon} \operatorname{Op}^{\mathrm{w}}\left(F_{-}\left(a_{0}\right) \tilde{F}_{-}(b)\right) R(\zeta)^{m} k^{-t-m+1 / 2-\epsilon}\right\| \leq C, \\
& \left\|k^{-t-m+1 / 2-\epsilon} R(\zeta)^{m} \operatorname{Op}^{\mathrm{w}}\left(F_{-}\left(a_{0}\right) \tilde{F}_{+}(b)\right) k^{t-1 / 2-\epsilon}\right\| \leq C .
\end{aligned}
$$

(iv) Suppose $\tilde{F}_{+}$and $\tilde{F}_{-}$satisfy the assumptions from (iii), $F_{-}^{1}, F_{-}^{2} \in$ $C_{0}^{\infty}(\mathbb{R})$ and

$$
\operatorname{dist}\left(\operatorname{supp}\left(\tilde{F}_{+}\right), \operatorname{supp}\left(\tilde{F}_{-}\right)\right)>0 .
$$

Then for all $m \in \mathbb{N}$ and all $t>0$ there exists $C>0$ such that

$$
\left\|k^{t} \mathrm{Op}^{\mathrm{w}}\left(F_{-}^{1}\left(a_{0}\right) \tilde{F}_{-}(b)\right) R(\zeta)^{m} \mathrm{Op}^{\mathrm{w}}\left(F_{-}^{2}\left(a_{0}\right) \tilde{F}_{+}(b)\right) k^{t}\right\| \leq C .
$$

Suppose $F_{+}$is given as in (ii), some functions $\tilde{F}_{+}, \tilde{F}_{-}, F_{-}$are given as in (iii) and suppose $\operatorname{dist}\left(\operatorname{supp}\left(F_{-}\right), \operatorname{supp}\left(F_{+}\right)\right)>0$. Then for all $m \in \mathbb{N}$ and all $t>0$ there exists $C>0$ such that

$$
\begin{aligned}
& \left\|k^{t} \mathrm{Op}^{\mathrm{w}}\left(F_{+}\left(a_{0}\right)\right) R(\zeta)^{m} \mathrm{Op}^{\mathrm{w}}\left(F_{-}\left(a_{0}\right) \tilde{F}_{+}(b)\right) k^{t}\right\| \leq C, \\
& \left\|k^{t} \mathrm{Op}^{\mathrm{w}}\left(F_{-}\left(a_{0}\right) \tilde{F}_{-}(b)\right) R(\zeta)^{m} \mathrm{Op}^{\mathrm{w}}\left(F_{+}\left(a_{0}\right)\right) k^{t}\right\| \leq C .
\end{aligned}
$$

The proof of Theorem 3.1 reduces by elementary algebra to the case of bounds with only one resolvent i.e. $m=1$, cf. [10] or [9]. The partition of unity needed for this reduction is indicated in (4.7). In the case $m=1,(3.4 \mathrm{a})$ follows by a singular Mourre theory while (3.4d) and (3.4e) follow by a certain modification of a method of [7]. The bounds (3.4b) and (3.4c) may be thought of as energy localizations. Certain 
energy-dependent positivity bounds, given by the Fefferman-Phong inequality in a certain Hörmander-Weyl calculus, play an important role in the proof of the bounds in (ii), (iii) and (iv) for $m=1$.

\section{Perturbative argument}

Using Theorems 2.3 and 3.1 one may easily prove Theorems 1.1 and 1.2 by perturbative arguments. In this section we will show Theorem 1.1. Let us write for $\zeta \in \Gamma_{\theta}$

$$
R(\zeta)=(H-\zeta)^{-1}, R_{1}(\zeta)=\left(H_{1}-\zeta\right)^{-1} ; H_{1}=p^{2}+V_{1}
$$

We shall proceed perturbatively using

$$
R(\zeta)\left(I+V_{2} R_{1}(\zeta)\right)=R_{1}(\zeta) .
$$

First we notice that $R_{1}(\zeta)$ is uniformly Hölder continuous in $\Gamma_{\theta}$. If $s>3 / 2(1+\mu / 2)$ this follows from (3.4a) with $m=2$ (showing in fact Lipschitz continuity in this case). If $s \leq 3 / 2(1+\mu / 2)$ we may interpolate the bounds of (3.4a) with $m=1$ and $m=2$.

In particular $R_{1}^{+}=R_{1}(0+i 0)=\lim _{\zeta \rightarrow 0, \zeta \in \Gamma_{\theta}} R_{1}(\zeta)$ and $R_{1}^{-}=R_{1}(0-$ $i 0)=\lim _{\zeta \rightarrow 0, \zeta \in \Gamma_{\theta}} R_{1}(\bar{\zeta})$ are well-defined (in weighted spaces).

To show (1.5) (in the general case) it suffices to show that $\langle x\rangle^{s}(I+$ $\left.V_{2} R_{1}^{+}\right)\langle x\rangle^{-s}$ is invertible as an operator on $L^{2}\left(\mathbb{R}^{d}\right)$. This follows from (4.2), the standard limiting absorption principle for positive energies and absence of positive eigenvalues, cf. [16], [21] and [4, Section 6.5]. Since $\langle x\rangle^{s} V_{2} R_{1}^{+}\langle x\rangle^{-s}$ is compact it suffices to show that the equation

$$
\phi=-V_{2} R_{1}^{+} \phi,
$$

has no nonzero solution $\phi \in\langle x\rangle^{-s} L^{2}\left(\mathbb{R}^{d}\right)$. Let $\psi=R_{1}^{+} \phi\left(\in\langle x\rangle^{s} L^{2}\left(\mathbb{R}^{d}\right)\right)$. Then we have in the sense of distributions

$$
H \psi=0 \text { and } \mathrm{V}_{2} \psi=-\phi .
$$

Using that $\frac{R_{1}^{+}-R_{1}^{-}}{2 i} \geq 0$ we obtain from the calculation

$$
0=\Im\left\langle\psi, V_{2} \psi\right\rangle=-\Im\langle\psi, \phi\rangle=-\Im\left\langle R_{1}^{+} \phi, \phi\right\rangle=(2 i)^{-1}\left\langle\phi,\left(R_{1}^{+}-R_{1}^{-}\right) \phi\right\rangle,
$$

that

$$
\psi=R_{1}^{+} \phi=R_{1}^{-} \phi
$$

We claim that

$$
\psi \in L^{2}\left(\mathbb{R}^{d}\right) .
$$

We shall prove (4.6) using Theorem 3.1 in a bootstrap argument. (For a similar problem for the free Laplacian see the proof of $[1$, Theorem 3.3].) We pick a real-valued function $F_{+}$as in Theorem 3.1 (ii) such that $F_{+}(x)=1$ for $|x|>2 C_{0}$. Let $F_{-}=1-F_{+}$. Pick real-valued 
functions $\tilde{F}_{-}$and $\tilde{F}_{+}$as in Theorem 3.1 (iii) such that $\tilde{F}_{-}+\tilde{F}_{+}=1$. Then we decompose with the symbols $a_{0}$ and $b$ being defined as in (3.1) with $E=0$ in the expression (3.2) for $f$

$$
\psi=\mathrm{Op}^{\mathrm{w}}\left(F_{+}\left(a_{0}\right)\right) \psi+\mathrm{Op}^{\mathrm{w}}\left(F_{-}\left(a_{0}\right) \tilde{F}_{-}(b)\right) \psi+\mathrm{Op}^{\mathrm{w}}\left(F_{-}\left(a_{0}\right) \tilde{F}_{+}(b)\right) \psi .
$$

By (3.4b) and (3.4d) the first two terms on the right hand side of (4.7) belong to $\langle x\rangle^{s^{\prime}} L^{2}$ where (assuming here $\phi \in\langle x\rangle^{-s} L^{2}$ )

$$
s^{\prime}=\left(1+\frac{\mu}{2}\right)\left(-t+\frac{1}{2}+\epsilon\right) ; t=\frac{s}{1+\frac{\mu}{2}}-\frac{1}{2}-\epsilon .
$$

We notice that the bound (3.4e) for $m=1$ is equivalent to

$$
\left\|k^{t-1 / 2-\epsilon} \operatorname{Op}^{\mathrm{w}}\left(F_{-}\left(a_{0}\right) \tilde{F}_{+}(b)\right) R_{1}(\zeta)^{*} k^{-t-1 / 2-\epsilon}\right\| \leq C .
$$

Taking $\zeta \rightarrow 0$ in the sector $\Gamma_{\theta}$, (4.9) leads to

$$
\left\|k^{t-1 / 2-\epsilon} \operatorname{Op}^{\mathrm{w}}\left(F_{-}\left(a_{0}\right) \tilde{F}_{+}(b)\right) R_{1}^{-} k^{-t-1 / 2-\epsilon}\right\| \leq C,
$$

with the same convention for $a_{0}$ and $b$ as above. We use the representation $\psi=R_{1}^{-} \phi$ of (4.5) and apply (4.10), and conclude that also the third term on the right hand side of (4.7) belongs to $\langle x\rangle^{s^{\prime}} L^{2}$ with $s^{\prime}$ given by (4.8); so $\psi \in\langle x\rangle^{s^{\prime}} L^{2}$.

From this and (4.4) we learn that $\phi \in\langle x\rangle^{s^{\prime}-1-\frac{\mu}{2}-\delta} L^{2}=\langle x\rangle^{-s-\delta+(2+\mu) \epsilon} L^{2}$; so by taking $\epsilon<<(2+\mu)^{-1} \delta$ we improve the decay of $\phi$ by almost a factor $\langle x\rangle^{-\delta}$. Iterating this argument leads to $s^{\prime} \leq 0$ eventually. We have proved (4.6).

Combining Theorem 2.3 and (4.6) yields $\psi=\phi=0$, completing the proof of (1.5) in the general case. The Hölder continuity statement of Theorem 1.1 in the general case follows readily by using (4.2) and the known result for $R_{1}(\zeta)$.

\section{ACKNOWLEDGMENTS}

S. Fournais was supported by a grant from the Carlsberg Foundation (before 31.12.02) and by a Marie Curie Fellowship of the European Community Programme 'Improving the Human Research Potential and the Socio-Economic Knowledge Base' under contract number HPMFCT-2002-01822 (from 01.01.03).

E. Skibsted is (partially) supported by MaPhySto - A Network in Mathematical Physics and Stochastics, funded by The Danish National Research Foundation. 


\section{REFERENCES}

[1] S. Agmon, Spectral properties of Schrödinger operators and scattering theory, Ann. Scula Norm. Sup. Pisa 2, no. 2 (1975), 152-218.

[2] D. Bollé, F. Gesztesy and W. Schweiger, Scattering theory for long-range systems at threshold, J. Math. Phys. 26, no. 7 (1985), 1661-1674.

[3] H.L. Cycon and P.A. Perry, Local time-decay of high energy scattering states for the Schrödinger equation, Math. Z. 188 (1984), 125-142.

[4] J. Dereziński and C. Gérard, Scattering theory of classical and quantum $N$ particle systems, Texts and Monographs in Physics, Springer-Verlag, Berlin, 1997.

[5] S. Fournais and E. Skibsted, Zero energy asymptotics of the resolvent for a class of slowly decaying potentials, MaPhySto Preprint nr. 14 (2003).

[6] C. Gérard, Asymptotic completeness for 3-particle systems, Invent. Math. 114 (1993), 333-397.

[7] C. Gérard, H. Isozaki, and E. Skibsted, $N$-body resolvent estimates, J. Math. Soc. Japan 48, no. 1 (1996), 135-160.

[8] L. Hörmander, The analysis of linear partial differential operators. III, SpringerVerlag, Berlin, 1985.

[9] H. Isozaki, Differentiability of generalized Fourier transforms associated with Schrödinger operators, J. Math. Kyoto Univ. 25, no. 4 (1985), 789-806.

[10] A. Jensen, Propagation estimates for Schrödinger-type operators, Trans. Amer. Math. Soc. 291, no. 1 (1985), 129-144.

[11] A. Jensen, High energy resolvent estimates for generalized many-body Schrödinger operators, Publ. RIMS, Kyoto Univ. 25 (1989), 155-167.

[12] A. Jensen and T. Kato, Spectral properties of Schrödinger operators and timedecay of the wave functions, Duke Math. J. 46, no. 3 (1979), 583-611.

[13] A. Jensen and G. Nenciu, A unified approach to resolvent expansions at thresholds, Rev. Math. Physics, 13, no. 6 (2001), 717-754.

[14] D. Jerison and C.E. Kenig, Unique continuation and absence of positive eigenvalues for Schrödinger operators, Ann. of Math. (2) 121, no. 3 (1985),463-494, With an appendix by E. M. Stein.

[15] K. Kitada, Time-decay of the high energy part of the solution for a Schrödinger equation, J. Fac. Sci. Univ. Tokyo Sect. IA Math. 31, no. 1 (1984), 109-146.

[16] É. Mourre, Absence of singular continuous spectrum for certain selfadjoint operators, Comm. Math. Phys. 78, no. 3 (1980/81), 391-408.

[17] S. Nakamura, Low energy asymptotics for Schrödinger operators with slowly decreasing potentials, Comm. Math. Phys. 161, no. 1 (1994), 63-76.

[18] J. Rauch, Local decay of scattering solutions to Schrödinger's equation, Comm. Math. Phys. 61 (1978), 149-168.

[19] M. Reed and B. Simon, Methods of modern mathematical physics I-IV, Academic Press, 1972-78.

[20] E. Skibsted, Long-range scattering of three-body quantum systems: Asymptotic completeness, Invent. Math. 151 (2003), 65-99.

[21] H. Tamura, Principle of limiting absorption for $N$-body Schrödinger operators, Letters in Math. Phys. 17 (1989), 31-36.

[22] D.R. Yafaev, The low energy scattering for slowly decreasing potentials, Comm. Math. Phys. 85, no. 2 (1982), 177-196. 
Laboratoire de Mathématiques, Université Paris-Sud - Bât 425, F91405 Orsay Cedex, France, soeren.Fournais@Math.u-PSUd.Fr

University of Aarhus, Ny Munkegade, 8000 Aarhus C, Denmark, SKIBSTED@IMF.AU.DK 